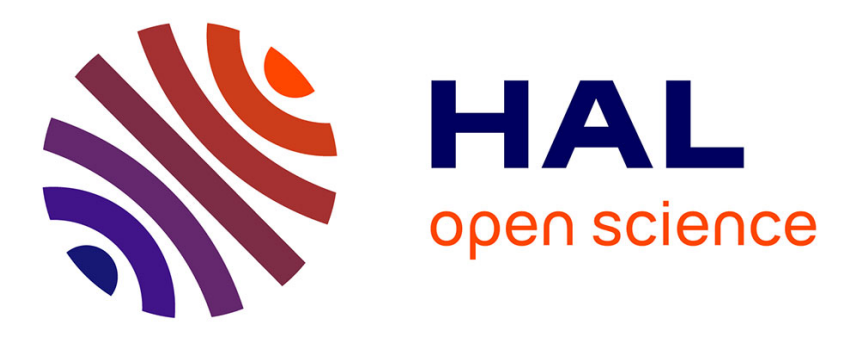

\title{
Isoprenoid biosynthesis in the diatom Haslea ostrearia
}

Anastasia Athanasakoglou, Emilia Grypioti, Sofia Michailidou, Codruta Ignea, Antonios M Makris, Kriton Kalantidis, Guillaume Massé, Anagnostis Argiriou, Frederic Verret, Sotirios Kampranis

\section{- To cite this version:}

Anastasia Athanasakoglou, Emilia Grypioti, Sofia Michailidou, Codruta Ignea, Antonios M Makris, et al.. Isoprenoid biosynthesis in the diatom Haslea ostrearia. New Phytologist, In press, 10.1111/nph.15586 . hal-01989337

\section{HAL Id: hal-01989337 https://hal.science/hal-01989337}

Submitted on 22 Jan 2019

HAL is a multi-disciplinary open access archive for the deposit and dissemination of scientific research documents, whether they are published or not. The documents may come from teaching and research institutions in France or abroad, or from public or private research centers.
L'archive ouverte pluridisciplinaire HAL, est destinée au dépôt et à la diffusion de documents scientifiques de niveau recherche, publiés ou non, émanant des établissements d'enseignement et de recherche français ou étrangers, des laboratoires publics ou privés. 
3 Anastasia Athanasakoglou ${ }^{1}$, Emilia Grypioti ${ }^{2}$, Sofia Michailidou ${ }^{3}$, Codruta Ignea ${ }^{1}$, Antonios M.

4 Makris $^{3}$, Kriton Kalantidis ${ }^{2,4}$, Guillaume Massé, ${ }^{5,6}$, Anagnostis Argiriou ${ }^{3}$, Frederic Verret ${ }^{2, *}$, Sotirios C.

$5 \quad$ Kampranis ${ }^{1, *}$

$6 \quad{ }^{1}$ Department of Plant and Environmental Sciences, University of Copenhagen, Thorvaldsensvej 40,

71871 Frederiksberg C, Denmark ${ }^{2}$ Department of Biology, University of Crete, P.O. Box 2208,

8 Heraklion 71003, Greece ${ }^{3}$ Institute of Applied Biosciences - Centre for Research and Technology

9 Hellas (INAB-CERTH), P.O. Box 60361, Thermi 57001, Thessaloniki, Greece ${ }^{4}$ Institute of Molecular

10 Biology and Biotechnology - Foundation of research and technology Hellas (IMBB-FORTH),

11 Heraklion, Greece ${ }^{5}$ UMI 3376 TAKUVIK, Centre national de la recherche scientifique (CNRS), France

$12 \quad{ }^{6}$ Département de Biologie, Université Laval, Québec, Canada

14 *Author for correspondence: Sotirios C. Kampranis, Department of Plant and Environmental Sciences,

15 University of Copenhagen, Thorvaldsensvej 40, 1871 Frederiksberg C, Denmark. Phone: +45 353329

1689 Email: soka@plen.ku.dk; Frederic Verret, Department of Biology, University of Crete, Heraklion,

17 Crete, Greece. Email: fverret@imbb.forth.gr.

18 


\section{Summary} isoprenoids.

-Summary: 176

- Diatoms are eukaryotic, unicellular algae that are responsible for about $20 \%$ of the Earth's primary production. Their dominance and success in contemporary oceans have prompted investigations on their distinctive metabolism and physiology. One metabolic pathway that remains largely unexplored in diatoms is isoprenoid biosynthesis, which is responsible for the production of numerous molecules with unique features.

- We selected the diatom species Haslea ostrearia because of its characteristic isoprenoid content and carried out a comprehensive transcriptomic analysis and functional characterization of the genes identified.

- We functionally characterized one farnesyl diphosphate synthase, two geranylgeranyl diphosphate synthases, one short-chain polyprenyl synthase, one bifunctional isopentenyl diphosphate isomerase - squalene synthase and one phytoene synthase. We inferred the phylogenetic origin of these genes and used a combination of functional analysis and subcellular localization predictions to propose their physiological roles.

- Our results provide insight into isoprenoid biosynthesis in H. ostrearia and propose a model of the central steps of the pathway. This model will facilitate the study of metabolic pathways of important isoprenoids in diatoms, including carotenoids, sterols and highly branched

Keywords: Heterokonts, isoprenoids, phytoene synthase, prenyltransferase, squalene synthase

Total word count (excluding summary, references and legends): 6088 
42 -Materials and Methods: 918

43 -Results: 3005

$44 \quad$-Discussion: 1482

45 - Acknowledgements: 84

46 Number of figures: 4 (all in colour)

47 Number of tables: 2

48 No of Supporting Information files: 1 (Methods S1, Table S1-Table S5, Fig. S1-Fig. S8, Notes S1-S2)

49

50 
Diatoms (phylum Heterokontophyta, class Bacillariophyceae) are one of the most diverse and ecologically important groups of phytoplankton. With more than 100,000 species that are widely distributed in aquatic environments, it is estimated that they contribute to about $20 \%$ of the global primary production. Consequently, they play central roles in aquatic food webs and in the biogeochemical cycling of nutrients (Nelson et al., 1995; Field et al., 1998; Falkowski, 2002). This profound ecological success has created great interest in distinctive physiological features of diatoms. Even though, several studies have already investigated unique facets of their metabolism (Kroth et al., 2008; Ast et al., 2009; Gruber et al., 2009; Allen et al., 2011; Fabris et al., 2012; Smith et al., 2012; Obata et al., 2013), there are still important gaps in our understanding of key biochemical pathways.

61 One such unexplored area is the biosynthesis of isoprenoids, a large class of metabolites that have vital biological functions in all domains of life (Holstein \& Hohl, 2004).

Despite their structural diversity, all isoprenoids are assembled from the same five-carbon atom precursors, isopentenyl diphosphate (IPP) and its isomer dimethylallyl diphosphate (DMAPP). Two distinct biosynthetic routes are responsible for the synthesis of these five-carbon precursors: the mevalonate (MVA) pathway and the methyl-erythritol-phosphate (MEP) pathway. Beyond these early serve as substrates for the synthesis of the different isoprenoid classes (geranyl diphosphate (GPP) for $\mathrm{C}_{10}$ monoterpenoids; farnesyl diphosphate (FPP) for $\mathrm{C}_{15}$ sesquiterpenoids, $\mathrm{C}_{30}$ triterpenoids and sterols; geranylgeranyl diphosphate (GGPP) for $\mathrm{C}_{20}$ diterpenoids and $\mathrm{C}_{40}$ carotenoids; etc.). This set of condensation reactions comprise the central steps of the pathway and are catalyzed by prenyltransferase-type enzymes. After this central part, synthesis of carotenoids and sterols proceeds through the commitment of FPP and GGPP to squalene and phytoene, respectively, by squalene/phytoene synthase-type enzymes (Fig. S1) (Vranová et al., 2012). 
Initial work using feeding experiments with labelled precursors and specific pathway inhibitors has shown that both the MVA and MEP pathways are present in diatoms (Cvejić \& Rohmer, 2000; Massé et al., 2004). Following the genomic sequencing of model diatom species Thalassiosira pseudonana (Armbrust et al., 2004) and Phaeodactylum tricornutum (Bowler et al., 2008), the focus of investigation has moved towards the final steps of the pathway, i.e. the carotenoid biosynthetic branch that provides important light harvesting and photoprotective molecules (Coesel et al., 2008; Dambek et al., 2012; Eilers et al., 2016) and the synthesis of sterols, which serve as membrane structural components (Fabris et al., 2014). An additional branch of isoprenoid biosynthesis that has attracted special interest is that of the highly branched isoprenoids (HBI). HBIs are only synthesized by a limited number of diatom species. They are extensively used as geochemical and paleoenvironmental markers (Massé et al., 2011; Belt \& Müller, 2013) and show potential for use as pharmaceuticals and as an alternative form of fuels (Rowland et al., 2001; Ferriols et al., 2015, 2017).

Nevertheless, the central steps of the isoprenoid pathway are less well explored and there is limited understanding on the function, subcellular localization and regulation of prenyltransferases from diatoms. Considering that the synthesis of prenyl diphosphates is a key regulatory step in the pathway that determines the flux towards different branches, a thorough investigation of the central steps will significantly improve our overall understanding of isoprenoid biosynthesis in diatoms. To this end, we selected the diatom species Haslea ostrearia for further studies, as this species is also able to synthesize HBIs (Wraige et al., 1999) (Fig. S2), thus providing a more comprehensive system to study isoprenoid biosynthesis. Through transcriptomic analysis, we in silico reconstructed the MVA and MEP pathways and confirmed the expression of five putative prenyltransferases and two putative phytoene/squalene synthases. We cloned six of these genes and characterized their function in a heterologous host or in vitro. By the combination of phylogenetic analysis, subcellular localization prediction and functional characterization, we proposed a model of the isoprenoid pathway in $H$. 
ostrearia. This model will serve as a basis for the elucidation of the biosynthesis of HBIs or other useful isoprenoids from diatoms.

\section{Materials and Methods}

\section{Diatom cultures}

The H. ostrearia clone NCC 153.8 used in this study was a product of heterothallic reproduction between clones NCC 141 and NCC 171, both of which were isolated in 2003

106 from natural populations of the oyster pond Lainard (La Barre de Monts $46^{\circ} 53^{\prime} 33^{\prime \prime} \mathrm{N}-02^{\circ} 07^{\prime}$ 107 59" W). Clone NCC 153.8 was kindly provided by Dr. Vona Medeler (University of Nantes) 108 and cultured at the University of Crete Greece, in $\mathrm{f} / 2$ medium (Guillard, 1975) at $20^{\circ} \mathrm{C}$ under 109 an irradiance of $50 \mu \mathrm{mol}$ photons $\mathrm{m}^{-2} \mathrm{~s}^{-1}(12 \mathrm{~h}: 12 \mathrm{~h}$ light:dark cycle) prior to analysis. The 110 ability of the strain NCC 153.8 to synthesize HBIs was confirmed by extraction and gas

111 chromarography-mass spectrometry GC-MS analysis (Methods S1, Fig. S3).

\section{$113 \quad$ RNA extraction and transcriptome sequencing}

114 Total RNA was extracted with Spectrum ${ }^{\mathrm{TM}}$ Plant Total RNA Kit (Sigma Aldrich, USA) and 115 quantified using the Qubit ${ }^{\mathrm{TM}}$ RNA BR Assay Kit (Invitrogen, Carlsbad, CA, USA). Messenger 116 RNA (mRNA) was isolated from total RNA using the NEBNext® Poly(A) mRNA Magnetic 117 Isolation Module (New England Biolabs). Complementary DNA (cDNA) library was 118 constructed using the NEBNext Ultra Directional RNA Library Kit for Illumina (New England 119 Biolabs), according to the manufacturer's instructions. Library quantification was conducted 120 with the KAPA Library Quantification kit for Illumina sequencing platforms (KAPA 121 BIOSYSTEMS, U.S.A.) on a Rotor-Gene Q thermocycler (Qiagen). Sequencing was 
122 performed at the Institute of Applied Biosciences of the Centre for Research and Technology

123 Hellas, on an Illumina NextSeq500 platform (Illumina, USA) using the NextSeq ${ }^{\mathrm{TM}}$ 500/550

124 Mid Output Kit (2 x 150 cycles) (Illumina, USA).

\section{Transcriptome Analysis}

The overall bioinformatics strategy included the following steps: (i) Trim and clean-up of the sequencing reads using the trim galore wrapper (https://github.com/FelixKrueger/TrimGalore) with default parameters, except for --length 40 and the --fastqc option, so as to remove adaptors and low-quality sequences; (ii) de novo assembly of the read using the Trinity software suite (Grabherr et al., 2011) with default parameters. All the analysis was implemented on a Linux/based HPC cluster assigning one node with 32 cores and 256 GB RAM.

\section{Gene identification, sequence and phylogenetic analysis}

Identification of candidate biosynthetic genes from $H$. ostrearia was based on homology with characterized and annotated homologues from red, green algae and heterokonts retrieved from the Genbank protein database (NCBI \& Resource, 2017) and DiatomCyc database (Fabris et al., 2012). For the phylogenetic analysis all diatom homologues were retrieved from the the Marine Microbial Eukaryote Transcriptome Sequencing Project (MMETSP) (Keeling et al., 2014) database using Blast search. The species names and corresponding MMETSP ID numbers are listed on Table S1. Sequences from other heterokonts, red algae, green algae, land plants, cyanobacteria, bacteria, archaea, fungi and metazoa were retrieved from GenBank protein database (NCBI \& Resource, 2017). Sequences were aligned using ClustalW (Larkin et al., 2007) and alignments were manually edited by exclusion of ambiguously aligned regions. Phylogenies were inferred using the maximum likelihood method 
146 (Whelan \& Goldman, 2001) and JTT matrix-based model in MEGA version 7 (Kumar et al., 2016). All

147 positions with less than $85 \%$ site coverage were eliminated. Branch support was generated using non-

148 parametric bootstrap analysis with 100 replicates. Conserved motifs in the selected sequences were

149 identified using NCBI Conserved Domain Search (Marchler-Bauer et al., 2015). Prediction of 150 subcellular localization was carried out through ASAFind (Gruber et al., 2015) and Hectar (Gschloessl 151 et al., 2008) in combination with SignalP 3.0 (Bendtsen et al., 2004) and TMHMM v. 2.0 (Krogh et al., 152 2001) for signal peptide and transmembrane domain prediction, respectively.

\section{Gene amplification and cloning}

155 Basic sequence analysis and design of primers (Table S2) was performed using CLC workbench 156 (QIAGEN). Total RNA was extracted using Trizol and cDNA was synthesized using SuperScript III 157 RT (Thermo) and DNAseI (Roche) treatment. Both full length and truncated variants of the selected 158 genes were PCR amplified using Phusion High-Fidelity DNA Polymerase (New England BioLabs) and 159 cDNA as template. PCR products were gel purified, A-overhangs were added using MyTaq 160 Polymerase (BIOLINE) and the products were subsequently cloned into vector pCRII-TOPO using 161 TOPO TA cloning kit (Thermo). After digestion with the appropriate restriction enzymes, the digests 162 were ligated to bacterial and yeast expression vectors. For bacterial expression, pRSET (N-terminal 163 6xHis tag) or pET102-His (C-terminal 6xHis tag) or pET102-Trx-His (N-terminal TRX and C-terminal 164 6xHis tag) plasmid backbones, digested with the same restriction enzymes were used. For yeast 165 expression, vectors pUTDH3myc, pWTDHmyc and pHTDH3myc (Ignea et al., 2012) were used. Final 166 constructs were verified by sequencing. Gene expression in bacteria and yeast and protein purification 167 protocols are described in Methods S1. 
For the characterization of the prenyltransferases $200 \mu$ enzymatic assays were carried out in glass 171 vials. The reaction mixture contained $10 \mathrm{mM}$ MOPS buffer ( $\mathrm{pH} 7.0), 5 \mathrm{mM} \mathrm{MgCl} 2,1 \mathrm{mM}$ DTT, 1 $172 \mathrm{mg} / \mathrm{ml}$ BSA, $100 \mu \mathrm{M}$ prenyl diphosphate substrate. The substrates used were: dimethylallyl 173 pyrophosphate (D4287, Sigma-Aldrich), isopentenyl diphosphate (I0503, Sigma-Aldrich), geranyl 174 diphosphate (G6772, Sigma-Aldrich), farnesyl diphosphate (F6892, Sigma-Aldrich), geranylgeranyl diphosphate (G6025, Sigma-Aldrich). The reactions were initiated by addition of $50 \mathrm{ng}$ of purified enzyme. After $16 \mathrm{~h}$ incubation at $25{ }^{\circ} \mathrm{C}$, the reactions were terminated by addition of equal volume of $1772 \mathrm{~N} \mathrm{HCl}$ in $83 \% \mathrm{EtOH}$ and after 20 min incubation they were neutralized with $0.14 \mathrm{~mL}$ of $10 \% \mathrm{NaOH}$. 178 The hydrolyzed diphosphates were extracted three times using $300 \mu$ of hexane. The hexane extracts 179 were concentrated to a final volume of $50 \mu \mathrm{l}$ and $1 \mu \mathrm{l}$ of each reaction was used for GC-MS analysis 180 (Methods S1). Individual compounds were identified by comparing their GC retention indices and mass 181 spectra with those of authentic standards.

\section{Results}

\section{Transcriptomic analysis and identification of candidate biosynthetic genes}

H. ostrearia NCC 153.8 strain was cultured and its ability to produce HBIs was confirmed (Fig. S3) prior to RNA extraction, library construction and sequencing on Illumina platform. After quality

186 filtering and trimming a total of 38631556 pair-end reads were de novo assembled into contigs using 187 the Trinity suite (Haas et al., 2013). A total of 45508 contigs were obtained.

188 We screened the assembled transcriptome to identify genes that putatively encode enzymes of the MVA and MEP pathways. Exploiting the high degree of sequence conservation among genes of isoprenoid biosynthesis, we based our screening on other characterized or annotated algal sequences

191 (See Materials and methods section). A pair-wise alignment between the queries and the contigs 
obtained from the transcriptome assembly was performed with the Massblast tool (Veríssimo et al., 193 2017). Through this analysis, we identified seven contigs corresponding to full-length protein 194 sequences with high similarities to enzymes that catalyze the seven steps of the MEP pathway. We additionally identified six contigs with homology to genes involved in the conversion of acetyl-CoA to IPP, through the MVA route. The last step of the MVA pathway involves the isomerization of IPP to DMAPP by isopentenyl diphosphate isomerase (IDI) (Berthelot et al., 2012). Searching for an IDI, we were only able to identify a contig that corresponds to a fusion of an IDI with a squalene synthase (HoIDI-SQS). Squalene synthases catalyze dimerization of two farnesyl diphosphate molecules to form squalene, the precursor of all sterols (Spanova \& Daum, 2011). It was not possible to identify independent transcripts of IDI or SQS in our sequencing data and we concluded that only the fusion of the two genes was expressed under the specific conditions.

We continued our analysis focusing on the central steps, which are catalyzed by prenyltransferase-type enzymes. Mining our assembled transcriptome, we were able to retrieve five contigs with similarity to annotated trans-prenyltransferases (PTS). These included one putative farnesyl pyrophosphate (FPP) synthase, sharing $57 \%$ sequence similarity at the amino acid level, with a functionally characterized homologue from Rhizosolenia setigera (Ferriols et al., 2015) (from now on referred to as HoPTS1) and four other putative polyprenyl diphosphate synthases (named HoPTS2-5) that exhibited similarities to other diatom and algal prenyltransferases (Fig. 1a, Table 1). We investigated whether this set of the five trans-prenyltransferases is conserved among different diatom species by examining the publicly available diatom transcriptomes in the Marine Microbial Eukaryote Transcriptome Sequencing Project (MMETSP) database (Keeling et al., 2014). Based on homology searches, HoPTS1, HoPTS2 and HoPTS4 orthologs are present in all diatom species investigated (a total of twenty-six species, representing both centric and pennate diatoms). An HoPTS5 ortholog seems to be missing only in one species (Skeletonema menzelii), while a HoPTS3 ortholog seems to be absent from seven diatom 
216 species (Table S3, Fig. S4). Among the measured transcripts, HoPTS5 showed significantly high 217 expression levels (Fig. S5).

218 Sequence analysis of the identified prenyltranserases, led to identification of conserved polyprenyl 219 synthase domains (Fig. 1b) and conserved motifs. Among them, the two aspartic acid-rich motifs $220 \operatorname{DDxx}(\mathrm{xx}) \mathrm{D}$ (First Aspartic acid-Rich Motif -FARM; and the Second Aspartic acid-Rich Motif 221 SARM), found in characterized prenyltransferases from all domains of life. These motifs are involved 222 in the binding of magnesium ions and are essential for prenyltransferase activity. The identification of 223 intact motifs in all five PTSs suggested that these genes likely encoded for active enzymes. Conserved 224 amino acids were also observed at positions 4 and 5 upstream of FARM. It has previously been shown 225 that these residues are involved in the regulation of the product chain length (Tarshis et al., 1996; 226 Wang \& Ohnuma, 1999; Liang et al., 2002). In HoPTS1, like in other characterized FPP synthases, this 227 region contains aromatic residues that are bulkier and block further chain elongation (Fig. S6a). By 228 contrast, smaller residues were observed at this region in the remaining four PTSs, suggesting that 229 these likely synthesize longer chain products (Fig. S6b). The prenyl diphosphates synthesized in the central steps are allocated to different branches of the pathway for the synthesis of final isoprenoid products, with sterol and carotenoid pathways being the main ones. Specific enzymes commit FPP and GGPP to these pathways. As described above, we 233 identified a squalene synthase, N-terminally fused to an IDI (HoIDI-SQS). In the absence of additional 234 SQS transcripts, this bi-functional protein could be involved in committing FPP to the synthesis of 235 sterols. We also identified a single transcript, termed HoPSY, which shares $72 \%$ similarity with a 236 characterized phytoene synthase from P. tricornutum (Dambek et al., 2012) and could catalyze the first 237 committed step of carotenoid biosynthesis in H. ostrearia. Both HoIDI-SQS and HoPSY were found to 238 contain the conserved phytoene/squalene synthase domain (Fig. 1b). 
241 Diatoms have a distinctive evolutionary history. Red and green algae, as well as glaucophytes evolved 242 after a primary endosymbiotic event, when a heterotrophic eukaryote engulfed a cyanobacterial cell 243 (McFadden, 2001; Rodríguez-Ezpeleta et al., 2005). Diatoms arose from secondary endosymbiosis, 244 during which a different heterotrophic eukaryote captured a cell of red algal origin (Yoon et al., 2004; 245 Prihoda et al., 2012). After incorporation, the engulfed cell was transformed into the plastid organelle 246 (Bhattacharya et al., 2007), lost its mitochondria and nucleus and genetic information was transferred 247 into the nucleus of the heterotrophic host, in a process termed endosymbiotic gene transfer (EGT) 248 (Timmis et al., 2004). The evolutionary linkage between diatoms and red algae is usually observed in 249 phylogenetic surveys (Armbrust et al., 2004; Bowler et al., 2008; Frommolt et al., 2008). However, 250 such surveys have also revealed a green phylogenetic signal. It has been proposed that this is due to a 251 cryptic endosymbiotic event that involved a green algal cell, which was later replaced by the red algal 252 endosymbiont (Moustafa et al., 2009; Chan et al., 2011). Alternatively, the green related genes could 253 have been acquired through repeated horizontal gene transfer (HGT) events from green algae, early 254 after the evolution of the first diatoms (Oborník \& Green, 2005; Dorrell \& Smith, 2011). This 255 multisourced genetic background has created unique, chimeric metabolic pathways that combine 256 features from multiple lineages.

257 To investigate how this is reflected to isoprenoid biosynthesis we inferred the phylogenetic origin for 258 each of the candidate biosynthetic genes. Three different possibilities were distinguished: (1) origin 259 from the secondary heterotrophic host (2) origin from the red algal endosymbiont and (3) origin from a 260 green algal cell, either through EGT or HGT.

261 It is evident that the MEP pathway genes can only have algal origin, as only the autotrophic cells 262 involved in the evolution of diatoms had the plastidial route of isoprenoid biosynthesis. Taking this into 263 account, we set out to investigate whether it was a red or a green algal cell that mediated each of the 
264 MEP pathway gene transfers to diatoms (Fig. S7). According to our analysis, 1-deoxy-D-xylulose 5265 phosphate synthase (DXS), 1-deoxy-D-xylulose 5-phosphate reductoisomerase (DXR) and 2-C-methylD-erythritol-4-phosphate-cytidylyltransferase (MCT) were transferred from the red algal 267 endosymbiont. This is evident from the highly supported clustering of diatom and red algal proteins. 268 By contrast, 4-diphosphocytidyl-2c-methyl-d-erythritol kinase (CMK) and (E)-4-hydroxy-3-methylbut269 2-enyl diphosphate synthases (HDS) genes were transferred from a green algal related cell. For the 270 remaining two, 2-C-methyl-D-erythritol 2,4-cyclodiphosphate synthase (MDS) and 271 hydroxymethylbutenyl diphosphate reductase (HDR), diatom proteins form well separated clades, in 272 distance from their algal homologues. This topology suggests that the corresponding genes have 273 diverged so that their origin cannot be traced.

274 Prenyltransferases and phytoene/squalene synthases could have been acquired from any of the involved 275 cells. The poorly supported branching patterns obtain for HoPTS1 and its homologues did not allow us 276 to trace its exact evolutionary origin. HoPTS3 clusters closely to prenyltransferases from opisthokonts 277 (fungi/metazoan), while other algal and cyanobacterial homologues are branching separately. This 278 topology suggests heterotrophic host origin. By contrast, the topologies obtained for rest of the 279 prenyltransferases, as well as the phytoene/squalene synthases are suggestive of algal origin. For 280 HoPTS5 and HoPSY this is the red algal endosymbiont, as diatom and red algal proteins form well281 supported branches together. The HoIDI-SQS gene is only conserved among heterokonts, haptophytes 282 and dinoflagellates, so it is likely that the fusion occurred in the common ancestor of these groups 283 (Davies et al., 2015). We investigated the evolutionary origin of each domain seperately and both for 284 IDI and SQS inferred phylogenies indicate green algal origin. While for SQS the corresponding cluster 285 is poorly supported, for IDI, branching is more robust (Fig. S8). HoPTS2 and HoPTS4 have also been 286 acquired from an algal cell, however, due to divergence it was not possible to pinpoint their exact 287 origin. 
288 This divergence could alternatively be explained by gene duplication events. To resolve this, we 289 examined three other diatom species (P. tricornutum, T. pseudonana and Fragilariopsis cylindrus) that 290 have their genomes sequenced. We obtained the homologues of the H. ostrearia genes with non291 resolved origin and we identified their localization in the respective genome. In all cases, those genes 292 are localized in different genomic regions (Table S4), indicating that they have been likely 293 independently acquired.

294 For genes of the MVA pathway see the extended discussion on the phylogenetic analysis that is 295 included in the Supporting Information (Notes S1).

\section{Prediction of subcellular localization}

To facilitate analysis of the physiological roles of the identified genes, we investigated the presence of signal/target peptides and obtained insight into the subcellular localization of the encoded proteins. The different endosymbiotic events provided diatoms with secondary plastids surrounded by four membranes (Kroth \& Strotmann, 1999). As a result, nuclear encoded diatom proteins targeted to the chloroplast contain a bipartite $\mathrm{N}$-terminal pre-sequence, consisting of a signal peptide with a conserved motif at its cleavage site (ASAFAP motif), followed by a chloroplast transit peptide. These presequences are essential for efficient transportation through all of the four membranes (Gruber et al., 2007). Two independent computational tools, ASAFind (Gruber et al., 2015) and HECTAR (Gschloessl et al., 2008), developed for identification of such pre-sequences were used. A different tool that is used to predict transmembrane domains in protein sequences (TMHMM Server v. 2.0) (Krogh $e t$ al., 2001) was included to further support this analysis. The results are summarized on Table S5. As expected, enzymes of the MEP pathway are predicted to be targeted to the chloroplast and those of the MVA to the cytosol. HoPTS1 was found to contain a Type II signal anchor and analysis with TMHMM 
suggested a strong possibility for the presence of transmembrane domain at the $\mathrm{N}$-terminal region.

312 Taken together, these results suggest that HoPTS1 is likely localized on the ER membrane. The ER 313 membrane is continuous with the outermost chloroplastic membrane, so the possibility that HoPTS1 is 314 related to the periplastic space (the space between the second and third outermost membranes) cannot 315 be ruled out. For HoPTS3 and HoIDI-SQS, no target or signal peptides were predicted, indicating 316 cytosolic localization. HoPTS2, HoPTS5 and HoPSY were predicted to be targeted to the chloroplast, 317 and HoPTS4 to the mitochondria.

\section{Functional characterization of prenyltransferases}

320 Since the subcellular compartmentalization analysis revealed the presence of signal/target peptides for 321 the majority of the identified prenyltransferases, both the full length and different truncated variants of 322 the enzymes (Table 2) were cloned into bacterial vectors, in-frame with a $\mathrm{C}$ - or $\mathrm{N}$ - terminal His-tag. 323 This allowed the purification of the expressed proteins and the assessment of their activity in in vitro 324 enzymatic reactions with prenyl diphosphate substrates. IPP was always used as the homoallylic 325 substrate, while DMAPP, GPP, FPP and GGPP were used as the allylic substrates. Control reactions 326 with cells transformed with empty vectors and subjected to the same purification steps were ran in 327 parallel. Following acid hydrolysis that facilitated the removal of diphosphates from the substrates and 328 products, all reactions were extracted and analyzed by GC-MS. Compound identification was based on 329 combination of accurate mass and retention time comparisons between reaction products and acid 330 hydrolysis products of authentic standards.

331 To characterize HoPTS1, the full-length enzyme was initially incubated with DMAPP or GPP and IPP. 332 As no activity was detected, two truncated variants, HoPTS1(Ser73-end) and HoPTS1(Val89-end), 333 were tested with the same substrates. Analysis of the reaction extracts resulted in the identification of 
334 linalool (LOH) and nerolidol (NOH), the acid hydrolysis products of GPP and FPP, respectively. (Fig. 335 2a). In the presence of DMAPP and IPP both alcohols were detected, while in the presence of GPP and 336 IPP, NOH was the only product identified. No other substrate combination resulted in new product 337 formation, demonstrating that HoPTS1 is an FPP synthase.

338 Initial expression of full length and truncated versions of HoPTS2 resulted in formation of inclusion 339 bodies. To obtain soluble proteins the variants were subcloned in frame with thioreodoxin at the N340 terminus and a C-terminal 6xHis-tag. Soluble HoPTS2 fusions were obtained and purified. The 341 activities of the full-length enzyme and HoPTS2(Gly140-end) variant were tested with all substrate 342 combinations but no new product was formed. However, variant HoPTS2(Arg45-end) was active and 343 gave a range of $\mathrm{C}_{20}-\mathrm{C}_{30}$ prenyl diphosphates in the presence of $\mathrm{FPP}$ and IPP and $\mathrm{C}_{25}-\mathrm{C}_{30}$ prenyl 344 diphosphates when GGPP and IPP were used as reaction substrates. We were able to identify these 345 products only after hydrolysis treatment and formation of the corresponding prenyl alcohols (Fig. 2b). 346 The fact that the variant HoPTS2(Gly140-end) was inactive indicates the presence of a functionally 347 important region between amino acids 45 and 140. Taken together, these results suggest that $H o$ PTS2 348 is likely a short chain polyprenyl synthase.

349 As there was no prediction for the presence of any signal/target peptide in HoPTS3, the full-length 350 enzyme was expressed, purified and tested with different substrates. In presence of DMAPP and IPP, 351 there was no formation of new products. However, when GPP and IPP were used as reaction substrates, 352 GGPP was produced, detected as its acid hydrolysis product geranyl linalool (GGOH). The same 353 product was formed when the enzyme was incubated with FPP and IPP (Fig. 2c). These results, in 354 combination with the lack of a signal peptide predicted for HoPTS3, suggest that HoPTS3 is possibly a 355 cytosolic GGPP synthase in H. ostrearia. 
Efforts to characterize HoPTS4 were unsuccessful, as there was no activity detected in any reaction 358 extract analyzed, either when using the full-length protein or the truncated variant HoPTS4(Leu121359 end). The prediction of a mitochondrial target peptide at the $\mathrm{N}$-terminal region suggests that this, 360 possibly, affects the catalytic activity of HoPTS4 and more truncated variants should be tested to 361 identify the optimal cleavage position for obtaining an active enzyme.

362 Finally, full length HoPTS5 and one truncated variant, HoPTS5(Ser36-end), were tested with different 363 substrate combinations. Even though the full-length enzyme was inactive, the truncated variant HoPTS5(Ser36-end) produced GGPP as the main product. This was detectable by the formation of GLOH after acid hydrolysis of the reaction products. Minor peaks of $\mathrm{LOH}$ and NOH were detected in when DMAPP and IPP were used as substrates while NOH could be detected when GPP and IPP were used instead, indicating that the formation of GGPP proceeds via GPP and FPP (Fig. 2d). Taken together with the prediction of a chloroplastic target peptide in its $\mathrm{N}$-terminus, these results suggest that HoPTS5 possibly encodes for a GGPP synthase, likely functioning in the chloroplast providing the substrates for carotenoid and phytol biosynthesis.

\section{Functional characterization of the squalene/phytoene synthase family members}

373 IDI and SQS are enzymes that catalyze two non-consecutive reactions. IDI isomerizes IPP to DMAPP, 374 while SQS catalyzes the formation of squalene using two FPP molecules. In order to characterize the 375 HoIDISQS fusion, we studied the two domains separately. Initially, we evaluated the functionality of the SQS domain by introducing the full-length gene into a Saccharomyces cerevisiae strain engineered

377 to produce high amounts of FPP (Ignea et al., 2012). Analysis of the non-saponifiable lipid extract of yeast cells expressing the fusion showed production of higher amounts of squalene when HoIDISQS was expressed, comparing to the levels of squalene produced by the endogenous yeast squalene 
synthase (Fig. 3a). This suggests that the SQS domain of HoIDISQS likely encodes for a functional synthase.

The functionality of the IDI domain was assessed both in the fusion (HoIDISQS) and as a separately cloned IDI domain (HoIDI). These two enzymes were coexpressed with a monoterpene synthase from Salvia fruticosa, 1,8-cineole synthase (SfCinS1) (Kampranis et al., 2007), in a yeast strain engineered to produce GPP (Ignea et al., 2012). By catalyzing the isomerization of IPP to DMAPP, IDI provides substrates to the endogenous yeast enzyme, Erg20p, which produces GPP. It has been shown that functional IDI expression in this strain increased synthesis of 1,8-cineole by SfCinS1 (Ignea et al., 2011). We used the endogenous yeast IDI (ScIDI) as positive control. We sampled the head space of all yeast cell cultures expressing the different combinations with solid-phase microextraction (SPME). Their analysis showed that when HoIDISQS, HoIDI or ScIDI were expressed, significantly higher amounts of 1,8-cineole were produced (Fig. 3a), indicating that the IDI domain is functional both individually and in the fusion.

We also employed the yeast expression system for the characterization of the candidate phytoene synthase, HoPSY. During our signal/target peptide analysis, HoPSY was predicted to have a chloroplastic signal peptide. Taking into account the cleavage position predictions, we introduced the truncated variant $\mathrm{HoPSY}(\mathrm{Ser} 78$-end) into a yeast expression vector. In order to evaluate the 397 functionality of the variant, we employed an in vivo chromogenic assay. In this assay, the candidate 398 phytoene synthase is coexpressed with a GGPP synthase and a phytoene desaturase. The GGPP 399 synthase provides the substrate for phytoene biosynthesis. If the examined enzyme is functional and 400 produces phytoene, the desaturase will take it up to produce lycopene (Fig. 3c). As yeast colonies that 401 accumulate lycopene become orange colored, this assay provides a quick and reliable means for the 402 evaluation of phytoene synthase activity. We used the yeast strain AM94, which is engineered for the 403 efficient production of isoprenoids (Ignea et al., 2012), and introduced HoPSY(Ser78-end) together 
404 with Erg20p(Y95A), an engineered GGPP synthase (Ignea et al., 2015), and crtI, a phytoene desaturase 405 from Xanthophyllomyces dendrorhous (Verwaal et al., 2007). While control cells that were 406 transformed with an empty vector, instead of HoPSY(Ser78-end), did not develop any color, 407 coexpression of the three genes resulted in orange colored yeast colonies, confirming the ability of 408 HoPSY to synthesize phytoene (Fig. 3b).

\section{Discussion}

411 Aiming to shed light to isoprenoid biosynthesis in diatoms, we investigated different aspects of the 412 pathway in the species $H$. ostrearia. By combining transcriptomic analysis with functional 413 characterization of enzyme activities and predictions for the subcellular localization of the 414 corresponding proteins, we can propose a model of isoprenoid biosynthesis in $H$. ostrearia that is 415 summarized in Fig. 4 and discussed below.

H. ostrearia retains a functional cytosolic MVA pathway and a functional plastidial MEP pathway. The 417 majority of the sequenced diatom species investigated to date, have both routes for IPP and DMAPP 418 synthesis. However, these precursors seem to be differentially allocated towards the final isoprenoid 419 products across different species. A plant-like dichotomy has been observed in P. tricornutum, 420 Nitzschia ovalis and R. setigera. Accordingly, sterols are synthesized using precursors from the MVA 421 route, while the biosynthesis of carotenoids and the diterpenoid phytol proceeds via MEP generated 422 precursors in the chloroplast (Cvejić \& Rohmer, 2000; Massé et al., 2004). On the contrary, $H$. 423 ostrearia uses chloroplast-derived precursors to synthesize its main sterol (24-ethylcholest-5-en-3-ol) 424 (Massé et al., 2004). Contribution of the MEP pathway to sterol (24-methyl-cholesta-5,24(28)-dien-3b425 ol) biosynthesis is also observed in the centric species $T$. pseudonana under fast growing, nitrogen426 replete culture conditions (Zhang et al., 2009). Similar differentiations were shown for HBI 
427 biosynthesis, with $R$. setigera incorporating $\mathrm{C}_{5}$ precursors derived from the MVA route and $H$. 428 ostrearia synthesizing HBIs with precursors generated via the MEP pathway (Massé et al., 2004). 429 These different patterns on precursor partitioning indicate different regulation mechanisms that in 430 addition to being responsive to external conditions (for example nutrient availability), also possibly 431 involve precursor transportation between cytosol and chloroplast. MVA and MEP pathway cross-talk 432 has already been reported in some plants (Bick \& Lange, 2003; Hemmerlin et al., 2003a). Even though 433 there are substantial differences between the organization of the primary plastids of plants and the 434 secondary plastids of diatoms, the four membranes of diatoms' plastids were previously shown to be 435 permeable through specific transporters (Ast et al., 2009).

436 The first key step towards prenyl diphosphate synthesis is the isomerization of IPP to DMAPP by IDI. 437 Photosynthetic heterokonts including diatoms and brown algae, haptophytes and dinoflagellates are 438 characterized by the expression of a bifunctional protein fusion between IDI and SQS that catalyzes the 439 dimerization of FPP towards sterol synthesis. In H. ostrearia this enzyme fusion does not appear to 440 have any target peptide, while it is predicted to contain a pair of transmembrane helices at the C441 terminal part, suggesting ER localization. No other contig encoding for an IDI could be identified in 442 our transcriptomic data. Analysis of thirty three diatom species by Ferriols and coworkers (2017) 443 (Ferriols et al., 2017) showed that all but one specific strain of $R$. setigera, express an IDI-SQS fusion 444 and 19 of them additionally express an independent IDI. Many of these fall within the same 445 phylogenetic clade with the independent IDI from $R$. setigera that contains a putative chloroplast 446 targeting peptide. A growing body of evidence recently supports the occurrence of alternative splicing 447 in diatoms (Rastogi et al., 2018). Thus, the possibility that diatoms that only contain the IDI-SQS 448 fusion gene may also produce an alternatively spliced form producing only the IDI protein cannot be 449 ruled out. 
450 The intermediate step between the reactions catalyzed by IDI and SQS is FPP synthesis and according 451 to our functional characterization is likely catalyzed by HoPTS1. Sequence analysis of HoPTS1 452 suggested the presence of a type II signal anchor. This is responsible to anchor the enzyme to a 453 membrane. It is reasonable to assume that this is the ER membrane, which is continuous with the 454 outermost chloroplastic membrane in diatoms. Since HoPTS1 and HoIDISQS catalyze consecutive 455 reactions, we can speculate that they might also physically interact, forming an enzymatic complex that 456 is localized at the ER. Previous studies showed that H. ostrearia's main sterol, 24-ethylcholest-5-en-3457 ol, incorporates precursors generated from the plastid localized MEP pathway (Massé et al., 2004). 458 Taken together, these results indicate again precursor transportation from the chloroplastic stroma to 459 the cytosol. The mechanism and the exact regulation of such events are unknown. Recently, a novel 460 isoprenoid regulatory mechanism that possibly involves precursor transportation between subcellular 461 compartments has been described in plants. According to this, a cytosolic isopentenyl phosphate kinase 462 (IPK) and specific Nudix hydrolases regulate IPP supply to the pathway by active phosphorylation463 dephosphorylation of IP/IPP. Perturbation of IP supply in Nicotiana tabacum was shown to affect both 464 MVA and MEP derived isopenoids, highlighting a correlation with precursor transportation (Henry et 465 al., 2015, 2018). Mining the transcriptome of $H$. ostrearia for similar genes, we were able to identify 466 homologues of IPK and Nudix hydrolase (candidate sequences can be found in Supporting Information 467 Notes S2) that are also present in transcriptomes of other sequenced diatom species. Whether a similar 468 regulatory mechanism is present in diatoms and how this controls IP/IPP supply and/or transportation 469 are open questions that remain to be answered.

470 Our model suggests that HoPTS5 acts in the plastids to generate the GGPP that is essential for 471 carotenoid synthesis. The high relative expression levels of HoPTS5 (Fig. S5) probably reflect the high 472 demand for plastidial isoprenoid biosynthesis (primarily GGPP-derived carotenoids and phytol) under 473 the specific growth conditions. The first committed step towards carotenoids is catalyzed by the 
phytoene synthase HoPSY. Both of these enzymes were acquired from the red algal endosymbiont, a

475 fact that corroborates their predicted targeting to the chloroplast. HoPTS2, which was also predicted to 476 have chloroplastic localization, showed activity as a short-chain poly prenyl synthase. The lack of 477 detailed information on the isoprenoid content of diatoms prevents us from assigning a role to HoPTS2.

478 It is possible that this enzyme synthesizes polyprenyl diphosphates or the corresponding alcohols in 479 vivo. In plants these compounds have been shown to be incorporated in thylakoid membranes and 480 modulate their fluidity, influencing the overall photosynthetic performance (Bajda et al., 2009; 481 Surmacz \& Swiezewska, 2011; Akhtar et al., 2017).

482 We propose that cytosolic isoprenoid biosynthesis is supported by precursors generated by the MVA 483 pathway and prenyl diphosphates synthesized by HoPTS3. Our analysis showed that this is probably 484 the only enzyme that is not conserved among different diatom species. In H. ostrearia, the HoPTS3 485 gene likely originates from the heterotrophic host of secondary endosymbiosis.

486 Although it was not possible to characterize the activity of HoPTS4, we presume that this enzyme 487 likely acts as a prenyl transferase in the mitochondria. As there was no other prenyltransferase 488 predicted to be targeted to mitochondria, HoPTS4 probably uses substrates transported from the cytosol 489 or chloroplast. Crosstalk between mitochondria and chloroplast in diatoms has been previously 490 proposed for other metabolic processes (Prihoda et al., 2012).

491 Even though the majority of prenyltransferases catalyze the linear condensation of prenyl diphosphates, 492 there are examples where these enzymes are involved in the synthesis of irregular, neither head-to-tail 493 nor head-to-head, isoprenoids (Rivera et al., 2001; Demissie et al., 2013). A characteristic example is 494 an FPPS in Artemisia tridentata that among other reactions catalyzes the head-to-middle linkage of two 495 DMAPP molecules to produce the branched isoprenoid, lavandulyl diphosphate (Hemmerlin et al., 496 2003b). It is thus likely that HBI biosynthesis also involves prenyltransferase-type enzymes. 497 Furthermore, different HBI producing diatom species were shown to use different precursors for the 
synthesis of these molecules (Massé et al., 2004), suggesting that the pathway is regulated differently among species. By providing here a model of the isoprenoid pathway in $H$. ostrearia that illustrates the subcellular distribution of different prenyltransferases we believe that we provide a basis for future HBI biosynthetic studies that will test the currently identified or different enzymes, substrates and/or conditions.

\section{Conclusions}

505 In conclusion, our investigation has significantly contributed to our understanding of isoprenoid 506 biosynthesis in diatoms. At least five prenyltransferases mediate the prenyl diphosphate synthesis and 507 provide substrates to downstream branches of the pathway. It is likely that precursors are transported 508 from the plastids to the cytosol. Further studies that will confirm the localization of the enzymes in vivo 509 and explore the regulatory mechanisms and crosstalk between the MVA and MEP pathways are 510 essential for a thorough elucidation of the mechanisms involved. Our phylogenetic analysis provided an 511 insight into the multisourced genetic background of diatoms that is reflected clearly on isoprenoid 512 biosynthesis. While there is still a general debate on the extent of the contribution of each lineage (red 513 and green) to the genomes of diatoms (Dagan \& Martin, 2009; Burki et al., 2012; Deschamps \& 514 Moreira, 2012), it is commonly accepted that these events have armed diatoms with the genetic 515 potential and the metabolic plasticity to succeed in contemporary oceans. Finally, our results provide 516 the blueprint for the elucidation of the biosynthetic pathways leading to unique diatom isoprenoids, 517 such as HBIs.

\section{Accession Numbers}

519 All sequences from $H$. ostrearia mentioned in this study have been submitted to the GenBank database 520 (www.ncbi.nlm.nih.gov) with accession numbers provided on Table 1. Transcriptomic data have been 
521 submitted to the European Nucleotide Archive (ENA) Database under experiment accession 522 ERX2834706 and Run accession ERR2827962.

\section{Acknowledgements}

524 The authors wish to thank Dr. David Ian Pattison for assistance in GC-MS sample running and analysis 525 and Dr. Vona Medeler, the scientific curator of the Nantes Culture Collection (NCC), for providing the 526 NCC 153.8 strain. We kindly acknowledge the financial support of the European Commission through 527 projects PIRSES-GA-2010-269294-BIOVADIA and RISE 734708 - GHaNA (to SCK, KK and GM) 528 and the Greek Secretariat of Research and Technology through project 11SYN_3_770 (to SCK, AMM 529 and AAr) that was co-financed by the European Regional Development Fund.

532 AA, EG, SM, FV, CI carried out experiments. SCK, FV, KK, AMM, AAr, GM designed experiments. 533 AA, EG, SM, CI, FV, SCK, AMM, GM, KK, AAr analyzed results. AA and SCK wrote the 534 manuscript. All authors have read and commented on the manuscript. 
543 Akhtar TA, Surowiecki P, Siekierska H, Kania M, Van Gelder K, Rea KA, Virta LKA, Vatta M, 544 Gawarecka K, Wojcik J, et al. 2017. Polyprenols Are Synthesized by a Plastidial cis -

545 Prenyltransferase and Influence Photosynthetic Performance. The Plant Cell 29: 1709-1725.

546 Allen AE, Dupont CL, Oborník M, Horák A, Nunes-Nesi A, McCrow JP, Zheng H, Johnson DA, 547 Hu H, Fernie AR, et al. 2011. Evolution and metabolic significance of the urea cycle in photosynthetic 548 diatoms. Nature 473: 203-207.

549 Armbrust EV, Berges JA, Bowler C, Green BR, Martinez D, Putnam NH, Zhou S, Allen AE, Apt 550 KE, Bechner M, et al. 2004. The genome of the diatom Thalassiosira Pseudonana: Ecology, evolution, 551 and metabolism. Science 306: 79-86. plastids depend on nucleotide import from the cytosol. Proceedings of the National Academy of Sciences 106: 3621-3626.

Bajda A, Konopka-Postupolska D, Krzymowska M, Hennig J, Skorupinska-Tudek K, Surmacz L, Wójcik J, Matysiak Z, Chojnacki T, Skorzynska-Polit E, et al. 2009. Role of polyisoprenoids in tobacco resistance against biotic stresses. Physiologia Plantarum 135: 351-364.

Belt ST, Müller J. 2013. The Arctic sea ice biomarker IP 25 : a review of current understanding, 559 recommendations for future research and applications in palaeo sea ice reconstructions. Quaternary 560 Science Reviews: 1-17.

561 Bendtsen JD, Nielsen H, Von Heijne G, Brunak S. 2004. Improved prediction of signal peptides: 562 SignalP 3.0. Journal of Molecular Biology 340: 783-795.

563 Berthelot K, Estevez Y, Deffieux A, Peruch F. 2012. Isopentenyl diphosphate isomerase: A 
checkpoint to isoprenoid biosynthesis. Biochimie 94: 1621-1634.

Bhattacharya D, Archibald JM, Weber APM, Reyes-Prieto A. 2007. How do endosymbionts become organelles? Understanding early events in plastid evolution. BioEssays 29: 1239-1246.

Bick JA, Lange BM. 2003. Metabolic cross talk between cytosolic and plastidial pathways of isoprenoid biosynthesis: Unidirectional transport of intermediates across the chloroplast envelope membrane. Archives of Biochemistry and Biophysics 415: 146-154.

\section{Bowler C, Allen AE, Badger JH, Grimwood J, Jabbari K, Kuo A, Maheswari U, Martens C,} Maumus F, Otillar RP, et al. 2008. The Phaeodactylum genome reveals the evolutionary history of diatom genomes. Nature 456: 239-244.

Burki F, Flegontov P, Oborník M, Cihlář J, Pain A, Lukeš J, Keeling PJ. 2012. Re-evaluating the green versus red signal in eukaryotes with secondary plastid of red algal origin. Genome Biology and Evolution 4: 626-635.

Chan CX, Reyes-Prieto A, Bhattacharya D. 2011. Red and green algal origin of diatom membrane transporters: Insights into environmental adaptation and cell evolution. PLOS ONE 6: e29138.

Coesel S, Oborník M, Varela J, Falciatore A, Bowler C. 2008. Evolutionary Origins and Functions of the Carotenoid Biosynthetic Pathway in Marine Diatoms. PLOS ONE 3: e2896.

Cvejić JH, Rohmer M. 2000. CO2as main carbon source for isoprenoid biosynthesis via the mevalonate-independent methylerythritol 4-phosphate route in the marine diatoms Phaeodactylum tricornutum and Nitzschia ovalis. Phytochemistry 53: 21-28.

Dagan T, Martin W. 2009. Seeing green and red in diatom genomes. Science 324: 1651-1652.

Dambek M, Eilers U, Breitenbach J, Steiger S, Büchel C, Sandmann G. 2012. Biosynthesis of fucoxanthin and diadinoxanthin and function of initial pathway genes in Phaeodactylum tricornutum. 
Davies FK, Jinkerson RE, Posewitz MC. 2015. Toward a photosynthetic microbial platform for terpenoid engineering. Photosynthesis Research 123: 265-284.

Demissie ZA, Erland LAE, Rheault MR, Mahmoud SS. 2013. The biosynthetic origin of irregular monoterpenes in lavandula: Isolation and biochemical characterization of a novel cis-prenyl diphosphate synthase gene, lavandulyl diphosphate synthase. Journal of Biological Chemistry $\mathbf{2 8 8}$ 6333-6341.

Deschamps P, Moreira D. 2012. Reevaluating the green contribution to diatom genomes. Genome Biology and Evolution 4: 683-688.

Dorrell RG, Smith AG. 2011. Do red and green make brown?: Perspectives on plastid acquisitions within chromalveolates. Eukaryotic Cell 10: 856-868.

Eilers U, Dietzel L, Breitenbach J, Büchel C, Sandmann G. 2016. Identification of genes coding for 598 functional zeaxanthin epoxidases in the diatom Phaeodactylum tricornutum. Journal of Plant 599 Physiology 192: 64-70.

Fabris M, Matthijs M, Carbonelle S, Moses T, Pollier J, Dasseville R, Baart GJE, Vyverman W, 601 Goossens A. 2014. Tracking the sterol biosynthesis pathway of the diatom Phaeodactylum tricornutum. 602 New phytologist 204: 521-535.

Fabris M, Matthijs M, Rombauts S, Vyverman W, Goossens A, Baart GJE. 2012. The metabolic blueprint of Phaeodactylum tricornutum reveals a eukaryotic Entner-Doudoroff glycolytic pathway. Plant Journal 70: 1004-1014.

606 Falkowski PG. 2002. The ocean's invisible forest. Scientific American 287: 54-61. 
S. 2017. An exception among diatoms: unique organization of genes involved in isoprenoid biosynthesis in Rhizosolenia setigera CCMP 1694. Plant Journal 92: 822-833.

Ferriols VMEN, Yaginuma R, Adachi M, Takada K, Matsunaga S, Okada S. 2015. Cloning and characterization of farnesyl pyrophosphate synthase from the highly branched isoprenoid producing diatom Rhizosolenia setigera. Scientific Reports 5: 10246.

Field CB, Behrenfeld MJ, Randerson JT, Falkowski P. 1998. Primary production of the biosphere: Integrating terrestrial and oceanic components. Science 281: 237-240.

\section{Frommolt R, Werner S, Paulsen H, Goss R, Wilhelm C, Zauner S, Maier UG, Grossman AR,} Bhattacharya D, Lohr M. 2008. Ancient recruitment by chromists of green algal genes encoding enzymes for carotenoid biosynthesis. Molecular Biology and Evolution 25: 2653-2667.

\section{Grabherr MG, Haas BJ, Yassour M, Levin JZ, Thompson DA, Amit I, Adiconis X, Fan L,} Raychowdhury R, Zeng Q, et al. 2011. Full-length transcriptome assembly from RNA-Seq data without a reference genome. Nature Biotechnology 29: 644-652.

Gruber A, Rocap G, Kroth PG, Armbrust EV, Mock T. 2015. Plastid proteome prediction for diatoms and other algae with secondary plastids of the red lineage. Plant Journal 81: 519-528.

Gruber A, Vugrinec S, Hempel F, Gould SB, Maier UG, Kroth PG. 2007. Protein targeting into complex diatom plastids: Functional characterisation of a specific targeting motif. Plant Molecular Biology 64: 519-530.

Gruber A, Weber T, Bartulos CR, Vugrinec S, Kroth PG. 2009. Intracellular distribution of the reductive and oxidative pentose phosphate pathways in two diatoms. Journal of Basic Microbiology 49: $58-72$.

Gschloessl B, Guermeur Y, Cock JM. 2008. HECTAR: A method to predict subcellular targeting in 
Haas BJ, Papanicolaou A, Yassour M, Grabherr M, Blood PD, Bowden J, Couger MB, Eccles D,

Li B, Lieber M, et al. 2013. De novo transcript sequence reconstruction from RNA-seq using the Trinity platform for reference generation and analysis. Nature Protocols 8: 1494.

\section{Hemmerlin A, Hoeffler JF, Meyer O, Tritsch D, Kagan IA, Grosdemange-Billiard C, Rohmer M,}

Bach TJ. 2003a. Cross-talk between the cytosolic mevalonate and the plastidial methylerythritol phosphate pathways in tobacco bright yellow-2 cells. Journal of Biological Chemistry 278: 2666663726676.

Hemmerlin A, Rivera SB, Erickson HK, Poulter CD. 2003b. Enzymes encoded by the farnesyl diphosphate synthase gene family in the Big Sagebrush Artemisia tridentata ssp. spiciformis. Journal of Biological Chemistry 278: 32132-32140.

Holstein SA, Hohl RJ. 2004. Isoprenoids: Remarkable diversity of form and function. Lipids 39: 293642309.

643 Ignea C, Cvetkovic I, Loupassaki S, Kefalas P, Johnson CB, Kampranis SC, Makris AM. 2011. 644 Improving yeast strains using recyclable integration cassettes, for the production of plant terpenoids. 645 Microbial Cell Factories 10: 4.

646 Ignea C, Trikka FA, Kourtzelis I, Argiriou A, Kanellis AK, Kampranis SC, Makris AM. 2012.

647 Positive genetic interactors of HMG2 identify a new set of genetic perturbations for improving 648 sesquiterpene production in Saccharomyces cerevisiae. Microbial cell factories 11: 162.

649 Ignea C, Trikka F a., Nikolaidis AK, Georgantea P, Ioannou E, Loupassaki S, Kefalas P, Kanellis 650 AK, Roussis V, Makris AM, et al. 2015. Efficient diterpene production in yeast by engineering 651 Erg20p into a geranylgeranyl diphosphate synthase. Metabolic Engineering 27: 65-75. 
JM, Degenhardt J, Makris AM, et al. 2007. Rational Conversion of Substrate and Product Specificity in a Salvia Monoterpene Synthase: Structural Insights into the Evolution of Terpene Synthase Function. The Plant Cell 19: 1994-2005.

\section{Keeling PJ, Burki F, Wilcox HM, Allam B, Allen EE, Amaral-Zettler LA, Armbrust EV,} Archibald JM, Bharti AK, Bell CJ, et al. 2014. The Marine Microbial Eukaryote Transcriptome Sequencing Project (MMETSP): Illuminating the Functional Diversity of Eukaryotic Life in the Oceans through Transcriptome Sequencing. PLoS Biology 12: e1001889.

Krogh A, Larsson B, von Heijne G, Sonnhammer ELL. 2001. Predicting transmembrane protein topology with a hidden Markov model: Application to complete genomes. Journal of molecular biology 305: 567-580.

\section{Kroth PG, Chiovitti A, Gruber A, Martin-Jezequel V, Mock T, Parker MS, Stanley MS, Kaplan}

A, Caron L, Weber T, et al. 2008. A model for carbohydrate metabolism in the diatom Phaeodactylum tricornutum deduced from comparative whole genome analysis. PLoS One 3: e1426.

Kroth P, Strotmann H. 1999. Diatom plastids: Secondary endocytobiosis, plastid genome and protein import. Physiologia Plantarum 107: 136-141.

Kumar S, Stecher G, Tamura K. 2016. MEGA7: Molecular Evolutionary Genetics Analysis Version 7.0 for Bigger Datasets. Molecular biology and evolution 33: 1870-1874.

\section{Larkin M, Blackshields G, Brown N, Chenna R, McGettigan P, McWilliam H, Valentin F,}

Wallace I, Wilm A, Lopez R, et al. 2007. ClustalW and ClustalX version 2. Bioinformatics 23: 29472948.

Liang PH, Ko TP, Wang AHJ. 2002. Structure, mechanism and function of prenyltransferases. European Journal of Biochemistry 269: 3339-3354. 


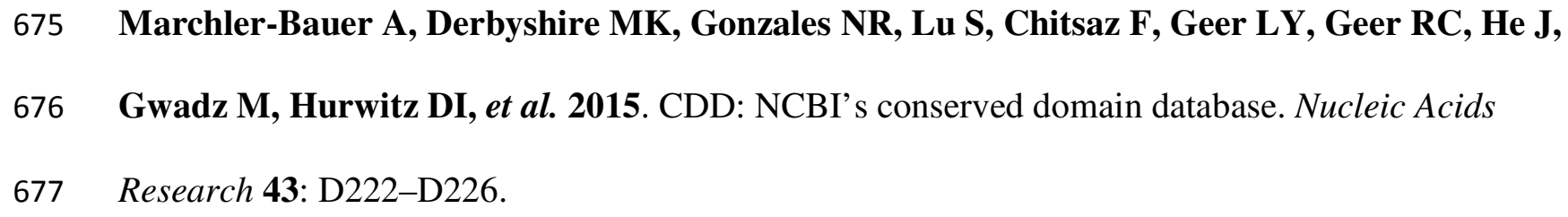


Prihoda J, Tanaka A, De Paula WBM, Allen JF, Tirichine L, Bowler C. 2012. Chloroplastmitochondria cross-talk in diatoms. Journal of Experimental Botany 63: 1543-1557. Scientific Reports 8: 4834.

\section{Rivera SB, Swedlund BD, King GJ, Bell RN, Hussey CE, Shattuck-Eidens DM, Wrobel WM,}

Peiser GD, Poulter CD. 2001. Chrysanthemyl diphosphate synthase: isolation of the gene and characterization of the recombinant non-head-to-tail monoterpene synthase from Chrysanthemum cinerariaefolium. Proceedings of the National Academy of Sciences of the United States of America 98: HJ, Philippe H, Lang BF. 2005. Monophyly of primary photosynthetic eukaryotes: Green plants, red algae, and glaucophytes. Current Biology 15: 1325-1330.

Rowland SJ, Belt ST, Wraige EJ, Massé G, Roussakis C, Robert JM. 2001. Effects of temperature on polyunsaturation in cytostatic lipids of Haslea ostrearia. Phytochemistry 56: 597-602. Smith SR, Abbriano RM, Hildebrand M. 2012. Comparative analysis of diatom genomes reveals Spanova M, Daum G. 2011. Squalene - biochemistry, molecular biology, process biotechnology, and applications. European Journal of Lipid Science and Technology 113: 1299-1320.

717 Surmacz L, Swiezewska E. 2011. Polyisoprenoids - Secondary metabolites or physiologically 718 important superlipids? Biochemical and Biophysical Research Communications 407: 627-632. 
Tarshis LC, Proteau PJ, Kellogg B a, Sacchettini JC, Poulter CD. 1996. Regulation of product chain length by isoprenyl diphosphate synthases. Proceedings of the National Academy of Sciences of the United States of America 93: 15018-15023.

Timmis JN, Ayliff MA, Huang CY, Martin W. 2004. Endosymbiotic gene transfer: Organelle genomes forge eukaryotic chromosomes. Nature Reviews Genetics 5: 123-135.

Veríssimo A, Bassard J-E, Julien-Laferrière A, Sagot M-F, Vinga S. 2017. MassBlast: A workflow to accelerate RNA-seq and DNA database analysis. bioRxiv.

\section{Verwaal R, Wang J, Meijnen JP, Visser H, Sandmann G, Van Den Berg JA, Van Ooyen AJJ.} 2007. High-level production of beta-carotene in Saccharomyces cerevisiae by successive transformation with carotenogenic genes from Xanthophyllomyces dendrorhous. Applied and Environmental Microbiology 73: 4342-4350.

Vranová E, Coman D, Gruissem W. 2012. Structure and Dynamics of the Isoprenoid Pathway Network. Molecular Plant 5: 318-333.

Wang K, Ohnuma S ichi. 1999. Chain-length determination mechanism of isoprenyl diphosphate synthases and implications for molecular evolution. Trends in Biochemical Sciences 24: 445-451.

Whelan S, Goldman N. 2001. A General Empirical Model of Protein Evolution Derived from Multiple Protein Families Using a Maximum-Likelihood Approach. Molecular Biology and Evolution 18: 691-699.

\section{Wraige EJ, Johns L, Belt ST, Mass $\sqrt{ } \subset$ G, Robert J-M, Rowland S. 1999. Highly branched C25} isoprenoids in axenic cultures ofHaslea ostrearia. Phytochemistry 51: 69-73.

Yoon HS, Hackett JD, Ciniglia C, Pinto G, Bhattacharya D. 2004. A Molecular Timeline for the Origin of Photosynthetic Eukaryotes. Molecular Biology and Evolution 21: 809-818. 
741 Zhang Z, Sachs JP, Marchetti A. 2009. Hydrogen isotope fractionation in freshwater and marine

742 algae: II. Temperature and nitrogen limited growth rate effects. Organic Geochemistry 40: 428-439.

743

744

745

746

747

748

749

750

751

752

753

754

755

756

757

758

759 
761 Additional supporting information may be found in the online version of this article.

762 Methods S1 Supplementary methods

Table S1 IDs of the transcriptomes from the MMETSP used in the phylogenetic analysis

Table S2 List of primers used in this study

Table S3 Identification of HoPTS1-HoPTS5 homologues in other diatom species

Table S4 Genomic location of isoprenoid biosynthetic genes in the P. tricornutum, T. pseudonana and F. cylindrus

Table S5 Summarized results from predictions of protein subcellular localization

Fig. S1 Overview of isoprenoid biosynthesis highlighting the different stages of the pathway (early, central, final steps)

Fig. S2 Chemical structures of some of the isoprenoids synthesized by H. ostrearia

Fig. S3 HBI profile of Haslea ostrearia NCC 153.8 strain

Fig. S4 Phylogenetic relationship of prenyltransferases from diatoms

Fig. S6 (a) Multiple sequence alignment of HoPTS1 with known farnesyl diphosphate synthases from other species (b) Multiple sequence alignment of HoPTS2-5 with known polyprenyl diphosphate synthases from other species

Fig. S7 Phylogenetic trees of MEP pathway proteins

Fig. S8 Phylogenetic trees of prenyltransferases and squalene/phytoene synthase family members 
Notes S1 Extended discussion on the phylogenetic analysis

781

Notes S2 Diatom sequences

782

783

784

785

786

787

788

789

790

791

792

793

794

795

796

797

798 
800 Table 1 Candidate isoprenoid biosynthetic genes, their closest homologues their evolutionary origin and 801 subcellular localization as indicated by analysis in this study

\begin{tabular}{|c|c|c|c|c|c|}
\hline & Gene Name & Genebank ID & $\begin{array}{l}\text { Closest homologue } \\
\text { (percentage similarity) }\end{array}$ & $\begin{array}{l}\text { Evolutionary } \\
\text { origin }\end{array}$ & $\begin{array}{c}\text { Subcellular localization } \\
\text { prediction }\end{array}$ \\
\hline \multirow{7}{*}{ 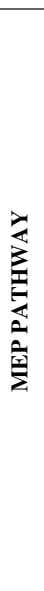 } & 1-deoxy-D-xylulose 5-phosphate synthase (DXS) & MH731010 & $\begin{array}{l}\text { Phaeodactylum tricornutum } \\
\text { XP_002176386.1 (83\%) }\end{array}$ & Red algae & Chloroplast \\
\hline & $\begin{array}{l}\text { 1-deoxy-D-xylulose 5-phosphate } \\
\text { reductoisomerase (DXR) }\end{array}$ & MH731011 & $\begin{array}{l}\text { Thalassiosira pseudonana XP_002295597.1 } \\
(84 \%)\end{array}$ & Red algae & Chloroplast \\
\hline & $\begin{array}{l}\text { 2-C-methyl-D-erythritol-4-phosphate- } \\
\text { cytidylyltransferase (MCT) }\end{array}$ & MH731012 & Fistulifera solaris GAX13480.1 (78\%) & Red algae & Chloroplast \\
\hline & $\begin{array}{l}\text { 4-diphosphocytidyl-2c-methyl-d-erythritol } \\
\text { kinase (CMK) }\end{array}$ & MH731013 & $\begin{array}{l}\text { Phaeodactylum tricornutum } \\
\text { XP_002178363.1 (77\%) }\end{array}$ & Green algae & Chloroplast \\
\hline & $\begin{array}{l}\text { 2-C-methyl-D-erythritol 2,4-cyclodiphosphate } \\
\text { synthase (MDS) }\end{array}$ & MH731014 & $\begin{array}{l}\text { Phaeodactylum tricornutum } \\
\text { XP_002180038.1 (76\%) }\end{array}$ & Algae & Chloroplast \\
\hline & $\begin{array}{l}\text { (E)-4-hydroxy-3-methylbut-2-enyl diphosphate } \\
\text { synthase (HDS) }\end{array}$ & MH731015 & $\begin{array}{l}\text { Fragilariopsis cylindrus OEU20628.1 } \\
(77 \%)\end{array}$ & Green algae & Chloroplast \\
\hline & $\begin{array}{l}\text { Hydroxymethylbutenyl diphosphate reductase } \\
\text { (HDR) }\end{array}$ & MH731016 & $\begin{array}{l}\text { Phaeodactylum tricornutum } \\
\text { XP_002178617 (73\%) }\end{array}$ & Algae & Chloroplast \\
\hline \multirow{7}{*}{ 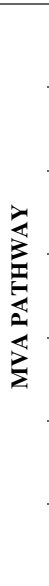 } & Acetyl-coa c-acetyltransferase 1 (AACT) & MH731017 & $\begin{array}{l}\text { Phaeodactylum tricornutum } \\
\text { XP_002185228.1 (69\%) }\end{array}$ & & Cytosol \\
\hline & Hydroxy-methylglutaryl-CoA synthase (HMGS) & MH731018 & $\begin{array}{l}\text { Fragilariopsis cylindrus OEU16767.1 } \\
(73 \%)\end{array}$ & & Cytosol \\
\hline & $\begin{array}{l}\text { Hydroxyl-methylglutaryl-CoA reductase } \\
\text { (HMGR) }\end{array}$ & MH731019 & $\begin{array}{l}\text { Fragilariopsis cylindrus OEU16221.1 } \\
(82 \%)\end{array}$ & & Cytosol \\
\hline & Mevalonate kinase (MVK) & MH731020 & $\begin{array}{l}\text { Thalassiosira pseudonana XP_002287787.1 } \\
(73 \%)\end{array}$ & & Cytosol \\
\hline & Phospho-mevalonate kinase (PMK) & MH731021 & $\begin{array}{l}\text { Fragilariopsis cylindrus OEU13939.1 } \\
(49 \%)\end{array}$ & & Cytosol \\
\hline & Mevalonate disphosphate decarboxylase (MVD) & MH731022 & $\begin{array}{l}\text { Fragilariopsis cylindrus OEU17781.1 } \\
(69 \%)\end{array}$ & & Cytosol \\
\hline & $\begin{array}{l}\text { Isopentenyl-diphosphate delta-isomerase fused to } \\
\text { squalene synthase (HoIDISQS) }\end{array}$ & MH720297 & Fistulifera solaris GAX27897.1 (63\%) & Green algae & Cytosol \\
\hline \multirow{6}{*}{ 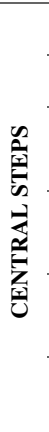 } & Farnesyl diphosphate synthase (HoPTS1) & MH720291 & Rhizosolenia setigera AKH49589.1 (56\%) & Not traced & ER or PPC \\
\hline & Polyprenyl diphosphate synthase (HoPTS2) & MH720292 & Thalassiosira oceanica EJK71722.1 (75\%) & Algae & Chloroplast \\
\hline & Geranylgeranyl diphosphate synthase ( $\mathrm{HoPTS} 3$ ) & MH720293 & $\begin{array}{l}\text { Phaeodactylum tricornutum } \\
\text { XP_002181666.1 (74\%) }\end{array}$ & $\begin{array}{l}\text { Heterotrophic } \\
\text { host }\end{array}$ & Cytosol \\
\hline & Putative polyprenyl synthase (HoPTS4) & MH720294 & $\begin{array}{l}\text { Phaeodactylum tricornutum } \\
\text { XP_002185039.1 (70\%) }\end{array}$ & Algae & Mitochondria \\
\hline & Geranylgeranyl diphosphate synthase (HoPTS5) & MH720295 & $\begin{array}{l}\text { Phaeodactylum tricornutum } \\
\text { XP_002178555.1 (75\%) }\end{array}$ & Red algae & Chloroplast \\
\hline & Phytoene synthase ( $\mathrm{HoPSY}$ ) & MH720296 & $\begin{array}{l}\text { Phaeodactylum tricornutum } \\
\text { XP_002178776.1 (69\%) }\end{array}$ & Red algae & Chloroplast \\
\hline
\end{tabular}

802 
Table 2 Functionally characterized enzymes in this study

805

806

807

808

809

810

811

812

813

Gly140-end

Full length no activity

\section{HoPTS2 1584 bp}

Arg45-end

FPP+IPP

C20-C30 PPP



Full length

GPP+IPP

FPP, GGPP

\begin{tabular}{|c|c|c|c|c|}
\hline & & & & \\
\hline & & & FPP+IPP & GGPP \\
\hline HoPTS4 & $1416 b p$ & Full length & no activity & - \\
\hline & & Leu121-end & no activity & \\
\hline HoTPS5 & $1011 \mathrm{bp}$ & Full length & no activity & - \\
\hline & & Ser36-end & DMAPP+IPP & GPP,FPP,GGPP \\
\hline & & & GPP+IPP & FPP, GGPP \\
\hline & & & FPP+IPP & GGPP \\
\hline HoPSY & $1485 \mathrm{bp}$ & Full length & Not tested & - \\
\hline & & Ser78-end & GGPP+GGPP & phytoene \\
\hline HoIDISQ & $2277 \mathrm{bp}$ & Full length & FPP+FPP & squalene \\
\hline
\end{tabular}


Fig. 1. (a) Neighbor joining phylogenetic tree of the selected sequences and their closest homologues and protein domain structures. Numbers on the branches indicate bootstrap support

(b) Protein domain structure of selected sequences. BSP; Bipartite Signal Peptide, MSP; Mitochondrial Signal Peptide, TD; Transmembrane domain.

Fig. 2. GC-MS profile of (a) HoPTS1(Ser73-end), (b) HoPTS2(Arg45-end), (c) HoPTS3, (d) HoPTS5(Ser36-end) in vitro reaction products using different prenyl diphosphates as substrates. Identification of compounds was based on comparison of accurate mass and retention time between reaction substrates/products and acid hydrolysis products of authentic standards (bottom panel). Ions $\mathrm{m} / \mathrm{z}$ 137, 205, 273, 341 and 409 are derived from precursor ions $(\mathrm{m} / \mathrm{z} 154,222,290,358$ and 426 respectively) by loss of $\mathrm{H}_{2} \mathrm{O}$ in positive ion mode

Fig. 3. Functional characterization of squalene/phytoene synthase family members. (a) Expression of $\mathrm{HoIDISQS}$ in the yeast strain AM94 resulted in high accumulation of squalene (up). Coexpression of HoIDISQS with SfCinS1 resulted in high accumulation of 1,8-cineole. Ho=Haslea ostrearia, $S f=$ Salvia fruticosa, $S c=$ Saccharomyces cerevisiae. (b) Yeast colonies coexpressing ERG20(Y95A), crtI and HoPSY(Ser78-end) (up) in comparison with the control colonies that carry an empty vector instead of a phytoene synthase (down) and (c) Lycopene biosynthetic pathway.

Fig. 4 A model for isoprenoid biosynthesis in $\boldsymbol{H}$. ostrearia. Isopentenyl diphosphate (IPP) and dimethyl allyl diphosphate (DMAPP) are synthesized via the mevalonate (MVA) and methylerythritol phosphate (MEP) pathways in the cytosol and plastid. Each of these precursor pools are used for the synthesis of prenyl diphosphates by prenyltransferases at different subcellular compartments. HoPTS1 
846 is responsible for farnesyl diphosphate (FPP) synthesis at the endoplasmic reticulum (ER), in close 847 proximity to $H o$ IDISQS. HoPTS3 mediates synthesis of geranylgeranyl diphosphate (GGPP) in the 848 cytosol. In the chloroplast HoPTS2 and HoPTS5 synthesize precursors for polyprenol and carotenoid 849 synthesis. The first committed step of carotenoid biosynthesis is catalyzed by HoPSY. HoPTS4 likely 850 mediates prenyl diphosphate synthesis in mitochondria. Dashed arrows indicate possible precursor 851 transportation between cytosol and plastids. 